\title{
PERFORMANCE OF RPL PROTOCOL BASED ON NEW OBJECTIVE FUNCTION FOR IOT- WSN
}

\author{
Zahraa A. Hamodi ${ }^{1}$, Abdulkareem A. Kadhim ${ }^{2}$ \\ 1,2 College of Information Engineering, Al-Nahrain University, Baghdad, Iraq \\ zahraaabd5966@gmail.com ${ }^{1}$, abdulkareem.a@ coie-nahrain.edu.iq ${ }^{2}$ \\ Received:5/4/2020, Accepted:16/5/2020
}

\begin{abstract}
The development of routing protocol to manage energy consumption of wireless sensor devices is one of the active research areas. The main challenge is the resource constraint (e. g. energy). In Internet of Things ( IoT), the devices operate in the presence of these limitations. Thus, an energy- efficient routing protocol is required. Routing Protocol for Low- Power and Lossy Network (RPL) is already introduced to enable IoT over Wireless Sensor Network. In this paper, a modified routing protocol depending on the maximum number of parent objective function ( MNP- OF) is proposed. MNP- OF uses a new node metric called Neighboring-metric ( Nmetric). The proposed protocol is called MNP based on Remaining Energy ( MRE) that combines N-metric with the node remaining energy metric for all nodes to the root node to select the preferred path. The results showed that MRE achieved up to $94 \%$ energy saving compared to RPL protocol in medium and large scale networks, while a reduction of about $33 \%$ in average remaining energy in small scale network. MRE showed considerable reductions in the average delay and the number of dead nodes combined with improvements in throughput, number of control messages, and number of switching parents for large scale network.
\end{abstract}

keywords: Routing protocols, WSN, IoT, RPL, Objective function.

\section{INTRODUCTION}

The WSNs applications are growing rapidly in recent years. This enabled the researchers to develop and improve WSN technology in different fields to satisfy the application requirements. WSN consists of tens to thousands of sensor nodes usually communicate through unreliable wireless channels over wide area, mainly for environmental monitoring. Once the environment changes, these tiny- sensors transmit data hop by hop to the sink node ( Gateway) via a routing protocol. In IoT, each thing operates on constraint devices called wireless sensor device ( node). By integrating WSN within IoT, the energy of these devices must be efficiently managed so that each device achieved the required application tasks/ goals within its alooted power. These tasks/ goals should be achieved without human intervention [1] . Managing the energy of all devices ( nodes) is considered as a big challenge in IoT as these devices are constrained in resources and capabilities ( e. g. , energy, bandwidth, and storage). Thus, energy- efficient routing protocol for constrained devices in IoT- network is required. In 2012, RPL [2] is standardized by Internet Engineering Task Force ( IETF) supporting two Objective Functions ( OFs); Objective Function Zero ( OF0) that uses Hop Count ( HC) node metric and the Minimum Rank with Hysteresis Objective Function ( MRHOF) that uses Expected Transmission Count ( ETX) as a link metric [2] , [3] . Research efforts in RPL routing protocol mainly focused on enhancing its performance by optimizing the existing routing metrics or developing new ones. Balancing the energy consumption of nodes in the network and implementing suitable routing metrics are the well known directions in researchs of RPL based routing protocols [4]. The efforts that involved the energy of the node with other metrics are summarized next. The authors in [5] used both the node remaining energy and existing traffic conditions to estimate the Expected Lifetime ( ELT) of the node. The results showed that the ELT metric performed better than ETX in energy consumption and worse than ETX in term of delay. Another work introduced in [6] , where the link metric ETX 
and node Energy metric are used. The results showed that, based on the energy objective function, the power consumption is decreased by about $28 \%$ and the stability ( number of switching parents) is lower than that of ETX by about $1.3 \%$ [6] . A new parent selection mechanism (uses ETX, expected transmission time ( ETT), and the remaining energy ( RE) of the node) is suggested to balance the workload among parents and to improve the lifetime of all meters in the network. An improvement in the Packet Reception Ratio ( PRR), the meters lifetime, and the parent workloads are achieved [7] . In Ref. [8], the authors used new metric that combines the energy consumption of a node with ETX for all paths to the root. The results showed that the proposed scheme performed better than RPL in terms of energy consumption, delay, PRR, and the number of dead nodes. When the number of nodes is increased, the performance of RPL and that of previously introduced protocols are degraded. In the present work, MNP based Remaining Energy ( MRE) routing protocol is proposed that uses both the $\mathrm{N}$ - metric and the node remaining energy in the parent selection process to improve the performance when the number of nodes is increased. In the present work, MNP based Remaining Energy ( MRE) routing protocol, both the Nmetric and the node remaining energy are involved in the parent selection process. The study here uses a novel approach where the number of control messages and switching parents in the network, in addition to other performance measures, are considered in the MRE routing protocol. The remaining parts of the paper are organized as follows. Section II describes the routing protocol for Low Power and Lossy Network ( LLNs). In Section III, the proposed protocol is presented and the simulation tests results are shown in Section VI. Section V covers the main concluding remarks of the work.

\section{Routing Protocol for Low Power and Lossy Network}

The routing algorithms or protocols can be classified based on different parameters as shown in Fig. 1 [9], [10] . The routing algorithms that depend on maintaining routing information ( routes establishment) can be classified into proactive, reactive, and hybrid routing protocols as shown in Fig. 1. The Proactive routing protocols always keep route information in the routing table at any time and used static network as the topology is fixed most of the time [9] . The standard RPL protocol is a proactive routing protocol used for LLNs application requirements. RPL constructs its Destination- Oriented Directed Acyclic Graphs ( DODAG) topology using an Objective Function ( OF) [3]. The objective function identifies the method used to construct one or more DODAG topology and how RPL nodes update routes and calculate ranks [11] . RPL OF identifies neighbors set, parents set, and preferred parents set nodes based on specified routing metrics and/ or constraints [11]. In RPL, the DODAG topology construction and maintenance are controlled by control messages ( ICMPv6 information messages) : DODAG Information Solicitation ( DIS), DODAG Information Object ( DIO), Destination Advertisement Object ( DAO), and Destination Advertisement Object Acknowledgment ( DAO- ACK) as shown in Fig. 2 DIO message is multicasted periodically with information about the topology. Fig. 3 shows the DIO message format [11]. Each DODAG identify itself by DODAG ID which is an IPv6 address ( 128- bits) given to DODAG root. The identified set of DODAGs is called a RPL Instance. Each RPL instance identifies itself by RPLinstanceID. The details about DIO message format can be found in RFC6550 [11]. RPL supports constraint- based routing applied to both the nodes and the links. If a node or a link does not satisfy a required constraint, it is pruned from the candidate neighbors set leading to a constrained shortest path as defined in RFC 6550 [11]. The set of supported link/ node metrics and constraints are specified in RFC 6551 [12].

https://ijict.edu.iq 


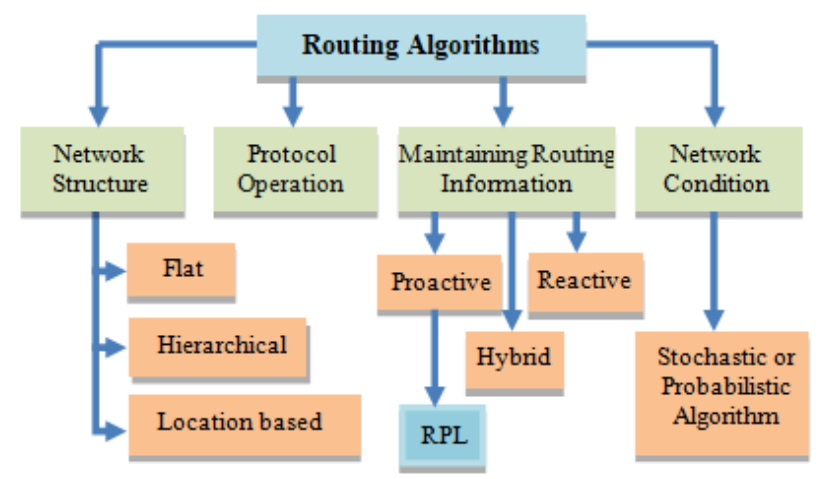

Figure 1: Classification of routing algorithms

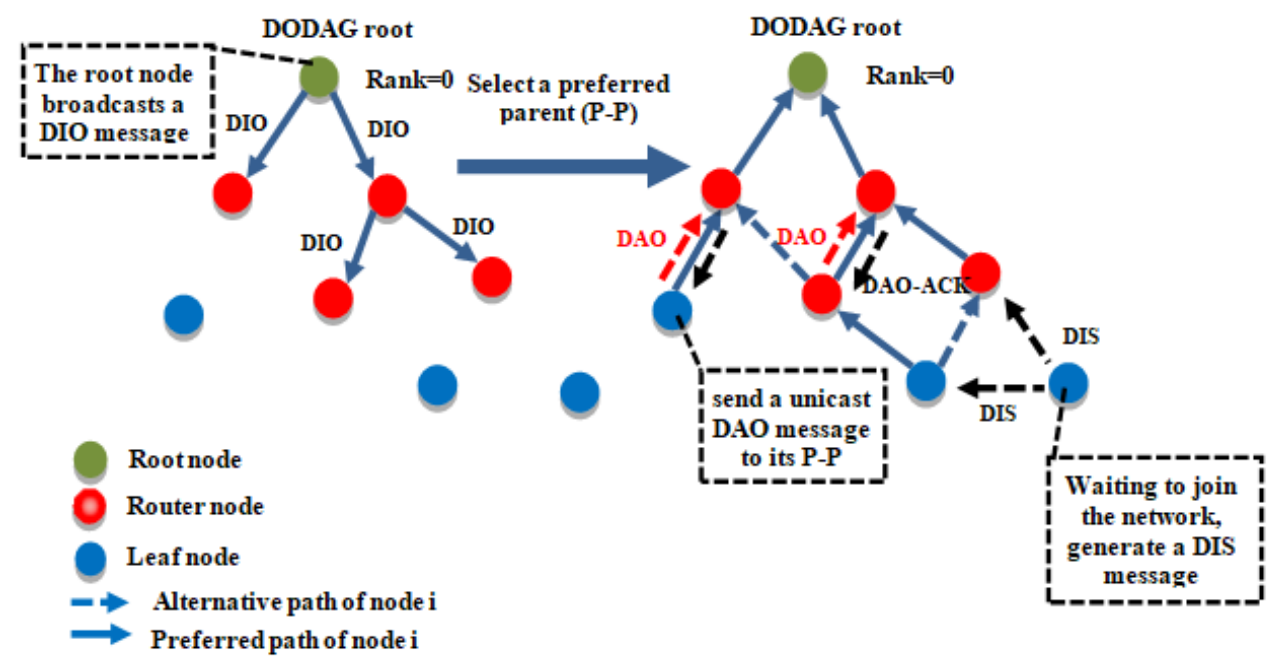

Figure 2: DODAG Construction

\begin{tabular}{|c|c|c|c|c|c|c|}
\hline \multicolumn{3}{|c|}{ 8-bits } & 8-bits & \multicolumn{2}{c|}{ 16-bits } \\
\hline G & 0 & $\begin{array}{c}\text { MOP } \\
\text { (3-bits) }\end{array}$ & $\begin{array}{c}\text { Pre } \\
\text { (3-bits) }\end{array}$ & Version Number & \multicolumn{2}{c|}{ Rank } \\
\hline \multicolumn{3}{|c|}{ (8-bits) } & $\begin{array}{c}\text { Flag } \\
\text { (8-bits) }\end{array}$ & $\begin{array}{c}\text { Reserved } \\
\text { (8-bits) }\end{array}$ \\
\hline \multicolumn{3}{|c|}{ DODAGID (128-bit) } \\
\hline \multicolumn{3}{|c|}{ Option (s)... } \\
\hline
\end{tabular}

Figure 3: DIO message format ( Re- drawn from [11])

\section{MNP Based Remaining Energy Protocol}

The proposed MNP based Remaining Energy Protocol ( MRE) selects the best route based on the Maximum Number of Parents objective function. This objective function uses N- metric which is the node metric that allow RPL to find the 
best path from the nodes to the root based on $\mathrm{N}$ - metric. The $\mathrm{N}$ - metric represents the number of parents for each neighbor node. In $\mathrm{N}$ - metric, the preferred parent ( $\mathrm{p}$ - $\mathrm{p}$ ) of a node is the neighbor node having maximum $\mathrm{N}$ - metric among available neighboring nodes. If there is no other neighbor node that has equal or more than the $\mathrm{N}$ - metric of $\mathrm{p}$ - $\mathrm{p}$, the node will not switch its $\mathrm{p}$ - $\mathrm{p}$ until the $\mathrm{p}-\mathrm{p}$ dies. MRE combined the N- metric with RE of each node called N- metric and RE metric ( $\mathrm{NE}$ ), where the node selects the path with maximum NE to be the preferred path to the root. Fig. 4 shows an example of path selection process in MRE. The mentioned N- metric represents the number of parents of each node. This $\mathrm{N}$ - metric usually changed if one of the neighboring nodes in the parent list dies and/ or the number of parents of the neighbor node is changed. The percentage of remaining energy associated to each node is an arbitrary value and used for demonstration only. As shown in Fig. 4, node- I has four paths to the root. The first two paths via node- F are I- F- B- A and I- F- C- A with $\mathrm{NE}$ values of $(2+8.8+1+7.9=19.7)$ and $(2+8.8+1+8.2=20)$, respectively. The other two paths via node- $\mathrm{G}$ are I- G- C- A and I- G- D- A with NE values of $(2+8.1+1+8.2=19.3)$ and $(2+8.1+1+8.6=19.7)$, respectively. Node- I will choose node-F as the preferred parent and the path I- F- C- A as the preferred path. If any node belonging to the preferred path consumes $1 \%$ of its energy, the NE value for this path will be changed and may become less than other paths. The node in this case needs to update the NE value for each path. This may result in changing the preferred path of the node.

\section{A. Operation of MRE}

MRE operates as in RPL, the root/ sink node multicasts DIO message to its neighboring nodes. DIO message contains the N-metric and RE of the node. The neighboring nodes are waiting for the DIO message to join the network. When a root neighboring node receives DIO message, it will directly select the root as a preferred parent ( $p$ - $p$ ) by sending back a DAO message. Then, it multicasts DIO message to its neighbors, including N- metric, RE, and NE value ( i.e combined value of RE metric and $\mathrm{N}$ - metric). If the DIO message is first received by the receiving node, the receiving node will directly select the sender node as p- p. If the DIO message from a new neighbor is received, it checks the NE value in the DIO input message and then it selects a new p- $\mathrm{p}$ node among the available parent candidates with the maximum $\mathrm{NE}$ value. Each node has one or more neighbors. For each neighbor, the node calculates the path metric ( NE value) and multicasts the new value to its neighbors. If two paths have the same NE value, the node will select the first path and keep the second one as an alternative path. The flow chart of MRE operation is shown in Fig. 5. If the node does not receive the DIO message, it will multicast DIS message to its neighbors. This is also happening when a new node wants to join the network. The receiver of DIS message will reset the trickle timer based on trickle timer algorithm and then multicast DIO to the requested node. The trickle timer mechanism is used by MRE to detect and respond to network instability and inconsistency. The instability includes: rank change of a node, detection of routing loops, and first time the node joining a DODAG. This mechanism is used by RPL DIO message to determine the frequency of sending messages in the network [13].

https://ijict.edu.iq 


\section{B. Metric calculation}

In the topology construction process of MRE, each node performs the following:

1) Determining the path metric for all neighbor nodes based on combined metric $N E$.

2) Frequently, updating the $N$ - metric of each neighbor in every time the node received DIO message.

If the node sends a message for the first time, the $\mathrm{N}$ - metric is given by the number of parents of each node. The node then updates its $\mathrm{N}$ - metric and sends it accordingly. The $N E$ value for each node is then calculated using the following equation:

$$
N E=N+R E
$$

Each node updates its NE value (called $N E_{n e w}$ ) and sends it within DIO message in the form of accumulated $N E$ value of the given path to the sender node as given by Eq. 2

$$
N E_{\text {path }}=\sum_{k=1}^{n}\left(N E_{n e w}\right)_{k}
$$

where $N E_{\text {path }}$ represents the accumulated value of $N E$ through the given path, $k$ is the number of nodes reside in this path, $n$ is the order of the last node in the path.

\section{Simulation Tests Results}

There are many real time operating systems exist to enable the implementation of IoT- WSN applications [14]- [17]. Contiki OS based on Cooja emulator is used in the work to implement and test the performance of the proposed protocol. Contiki OS is widely used searchers to implement WSN scenarios that supporting IoT [14]. The emulation parameters are shown in Table I. Different performance measures are used in the evaluation: the average remaining energy, the Packet Reception Ratio (PRR), the number of dead nodes, the average time delay, the throughput, the number of control messages, and the number of switching parents. The deployed network is used with different number of IoT motes/ nodes ( 20 , 40, and 80). The nodes are randomly distributed within the network coverage area. Three different areas of $100 \mathrm{~m} \times 100 \mathrm{~m}$, $150 \mathrm{~m} \times 150 \mathrm{~m}$, and $200 \mathrm{~m} \times 200 \mathrm{~m}$ are used. The type of mote/node used in this work is the Tmote sky [18] .The initial energy of all nodes is set to full energy of $3 \mathrm{~J}$ which is considered as $100 \%$. This value is assumed before the transmission is originated. Each node in the assumed scenarios transmits one data packet randomly in a period of one second. For each performance measure two conditions are considered in the tests: fixed number of nodes with variable areas and fixed area with variable number of nodes.

\section{A. The average remaining energy test}

For the fixed number of nodes test using different areas of $100 \times 100,150 \times 150$, and $200 \times 200 \mathrm{~m}^{2}$, the results are shown in Fig. 6, Fig. 7, and Fig. 8, for 20, 40, and 80 nodes, respectively. These figures show that, for 20 nodes RPL is better than MRE in terms of consumed average energy (i. e. RPL reserved more remaining energy) for all areas tested. For 40 and 80 nodes ( Fig. 7 and Fig. 8, respectively), the average remaining energy of MRE is more than that of RPL. Such improvement is about $7 \%$ for medium area $\left(150 \times 150 \mathrm{~m}^{2}\right)$ with 40 nodes. As mentioned previously, the main drawback 
of RPL is its degraded performance as the number of nodes is increased. For MRE, better performance is achieved due to the dependency on M- metric in the preferred parent selection. As the number of nodes increased, each node will have more than one neighboring nodes that are expected to be a preferred parent for such node. For the case of fixed area test, the results for $150 \mathrm{~m} \times 150 \mathrm{~m}$ using different number of nodes are shown in Fig. 9. It can be seen that MRE is better than RPL for the cases of 40 and 80 nodes, with improvement of $6 \%$ and $29 \%$, respectively, in the average remaining energy. There is no improvement in the case of 20 nodes since in this case each node has small number of neighboring nodes to serve as parents.

TABLE I

THE EMUlation PARAMETERS

\begin{tabular}{||c|c|}
\hline Parameter & Remarks of Values \\
\hline Deployment area & $100 \mathrm{~m} \times 100 \mathrm{~m}, 150 \mathrm{~m} \times 150 \mathrm{mand} 200 \mathrm{~m} \times 200 \mathrm{~m}$ \\
\hline Mote type & Tmote sky \\
\hline Node locations & Random \\
\hline Number of IoT motes & 20,40 and 80 \\
\hline Number of transmitted packets & 1 packet / second/ node \\
\hline Operating system & Contiki - V3.0 \\
\hline Simulation time & 60 minutes \\
\hline Simulator & Cooja based on C language \\
\hline Tested routing protocol & RPL and proposed MRE protocols \\
\hline Transmission range between nodes & $50 \mathrm{~m}$ \\
\hline
\end{tabular}

\section{B. The number of dead nodes results}

For the fixed number of nodes test using different areas of $100 \times 100,150 \times 150$, and $200 \times 200 \mathrm{~m}^{2}$, the results are shown in Fig. 10, Fig. 11, and Fig. 12, for 20, 40, and 80 nodes, respectively. These figures show that, for 20 nodes, MRE doubles the number of dead nodes in all areas when compared to RPL. This is because each node has small number of candidate parent nodes and these parent nodes maybe used by other nodes in the network as p- p, thus consume energy faster and die early. For 40 and 80 nodes (Fig. 11 and Fig. 12, respectively), the number of dead nodes decreased compared to RPL in all areas. Considering fixed area test, the results for $150 \mathrm{~m} \times 150 \mathrm{~m}$ using different number of nodes are shown in Fig. 13. For 20 nodes, RPL has lower number of dead nodes compared to MRE. When the number of nodes increased, the number of dead nodes in MRE is reduced by about $6 \%$ and $11 \%$ compared to RPL for 40 and 80 nodes, respectively.

\section{The average time delay results}

The average time delay is the average time taken to send the packet from the source node to its destination for all nodes. For the fixed number of nodes test using different areas of $100 \times 100,150 \times 150$, and $200 \times 200 \mathrm{~m}^{2}$, the results are shown in Fig. 14 for 40 nodes. In small area, it can be seen that the RPL showed higher average- time- delay compared to MRE. In small area, MRE shows less average delay compared to RPL by about $20 \%$, while the average delay is increased to about $22 \%$ in a medium area compared to RPL. RPL experienced higher delay compared to MRE by about $31 \%$ in a large area. Thus, MRE presented better average delay in large area. For the case of fixed area test, the results for $150 \mathrm{mX} 150 \mathrm{~m}$ using different number of nodes are shown in Fig. 15. When 20 nodes are used, MRE approximately showed the same delay as RPL. For 40 nodes, the average delay increased in MRE by about 22\% compared to RPL. While for the case of 80 nodes, RPL has higher average time delay compared to MRE by about 59\%. In RPL, the average time delay increased as the number of nodes is increased for fixed area. 


\section{Packet reception ratio results}

PRR is tested using different number of nodes for fixed area of $150 \mathrm{~m} \times 150 \mathrm{~m}$. Increasing the number of nodes usually affected PRR of the given protocols. Fig. 16 shows the PRR for $150 \mathrm{~m} \times 150 \mathrm{~m}$ area with different number of nodes ( 20, 40 and 80). MRE achived the same PRR as RPL in small and medium number of nodes. In large network, MRE showed better PRR than RPL by about 3\%. If the child messages did not reach the root due to intermediate nodes died, the PRR will be reduced.

\section{E. The throughput results}

The throughput represents the number of successful reception of message packets per second received by the destination node. Fig. 17 shows the throughput for network area of $150 \mathrm{~m} \times 150 \mathrm{~m}$. For 20 nodes, MRE achived the same throughput as RPL. While for 40 nodes, the throughput is decreased in MRE by about 10\% compared to RPL. When the number of nodes is increased to 80 , MRE achieved more throughput than RPL by about $46 \%$.

\section{F. The number of control messages results}

The number of control messages represents the number of ICMPv6 information messages that flow in the network during the test period. These messages are used to construct and maintain RPL DODAG topology. Also, the nodes exchange DIO and DAO messages in the parent selection and parent switching processes. Fig. 18 shows the number of control messages with different number of nodes for $150 \mathrm{~m} \times 150 \mathrm{~m}$ area. For MRE, the number of messages increased with the increase in the number of nodes. In all cases, MRE showed higher number of control messages compared to RPL. This is due to the use of frequent switching parents in MRE algorithm depending on the number of parent nodes and their remaining energy. RPL switch parents only in case of parent nodes died or when the link becomes bad. Also, the dependency on the same parent node by multiple child nodes resulting in re-transmissions that in turns increases the number of messages in the network.

\section{G. The Number of switching parents results}

Fig. 19 shows the number of switching parents for $150 \mathrm{~m} \times 150 \mathrm{~m}$ with different number of nodes. The Number of switching parents represents the number of times that child nodes change their parent nodes during the test period. For MRE, it can be seen that the number of switching parents increases as the network becomes denser compared to RPL. In general, the node switches its preferred parent to select the best energy- efficient or link quality parent to avoid the dead of parent node due to energy consumption or number of retransmissions when the link quality is bad. It is worth to mention that, the extra number of switching may increase the consumed energy, but this is relatively small compared to the consumed energy and so not covered by the present work.

https://ijict.edu.iq 


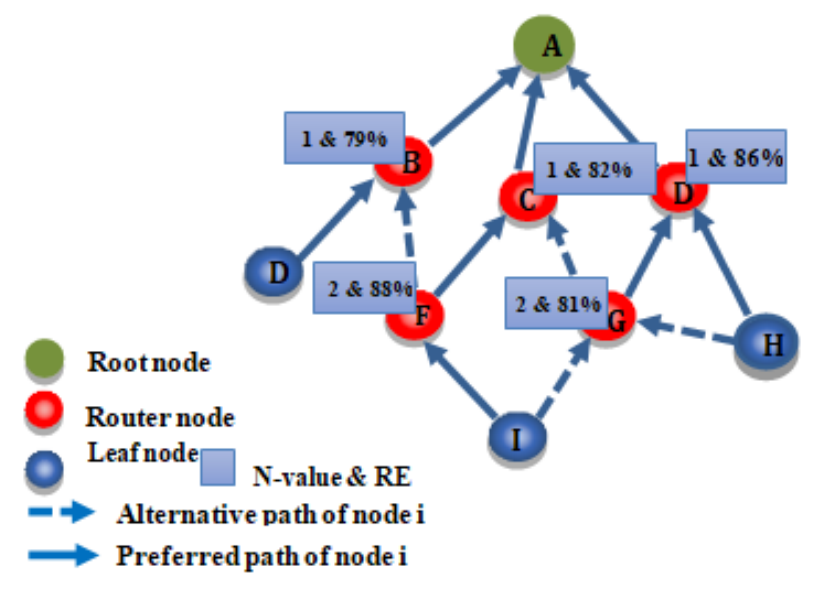

Figure 4: Path selection process in MRE protocol

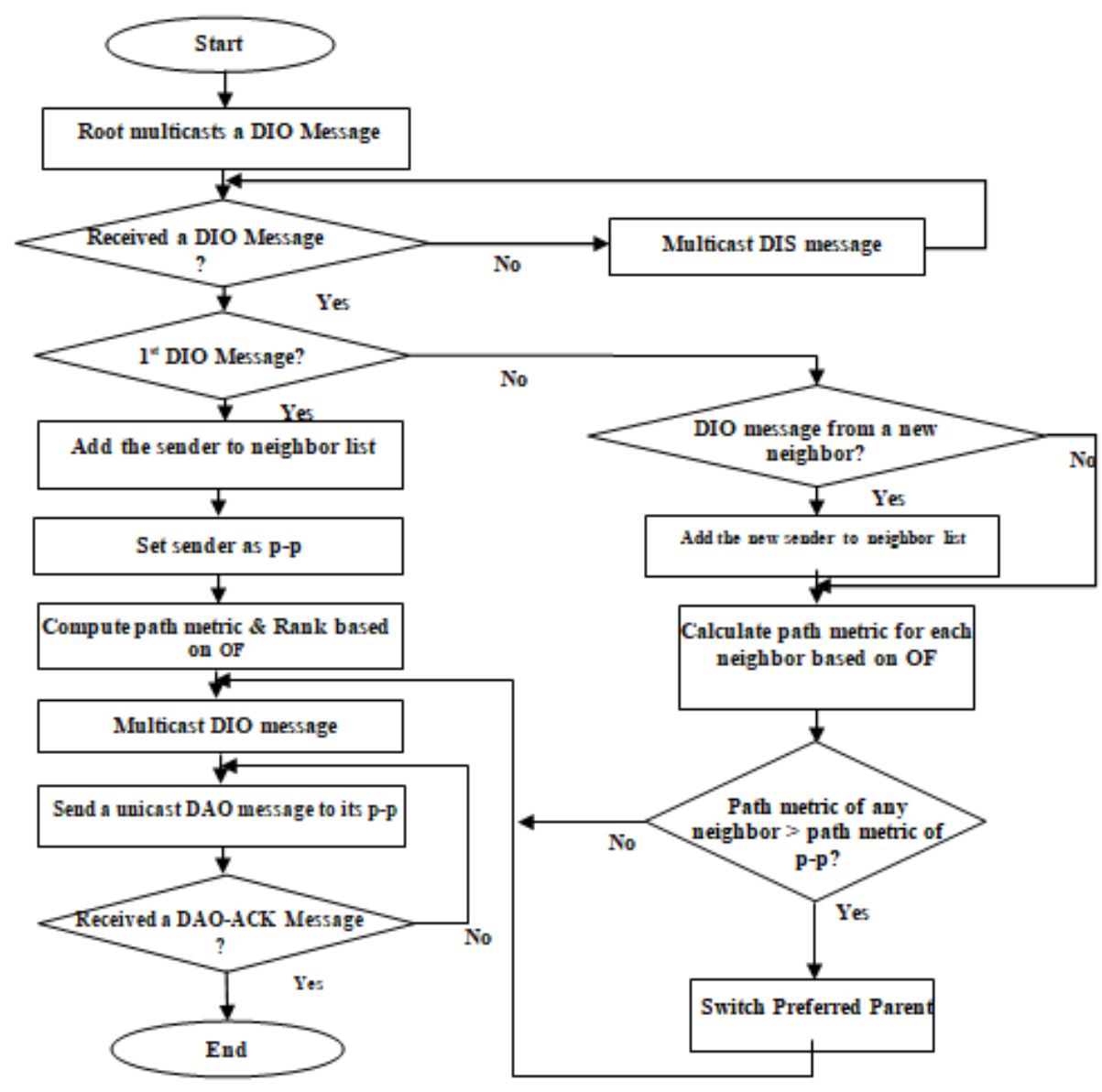

Figure 5: Flowchart of MRE protocol 


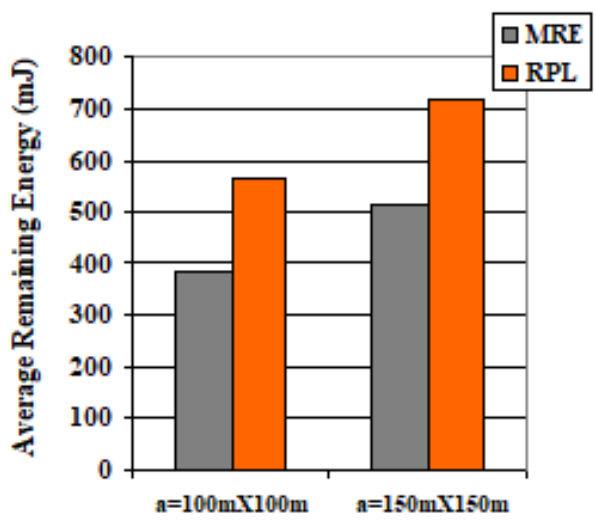

Figure 6: Average remaining energy for 20 nodes

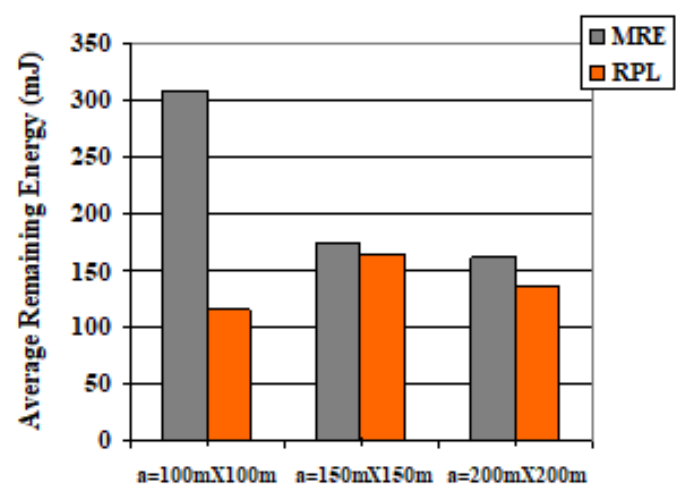

Figure 7: Average remaining energy for 40 nodes

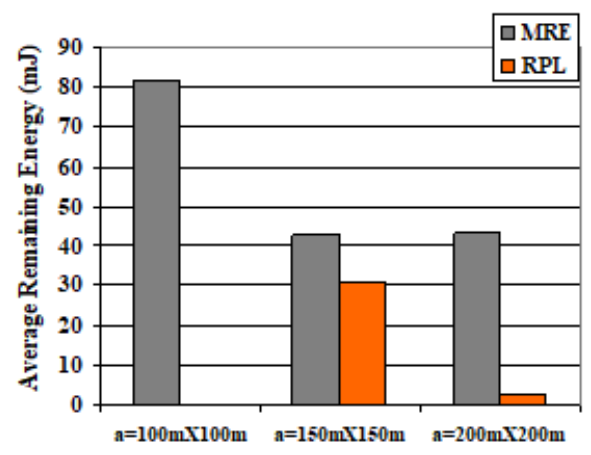

Figure 8: Average remaining energy for 80 nodes 


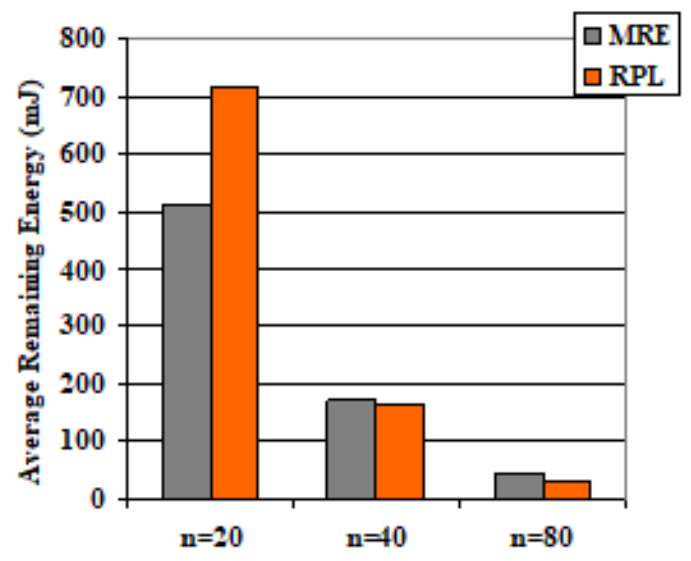

Figure 9: Average remaining energy for $150 \mathrm{~m} \times 150 \mathrm{~m}$ area

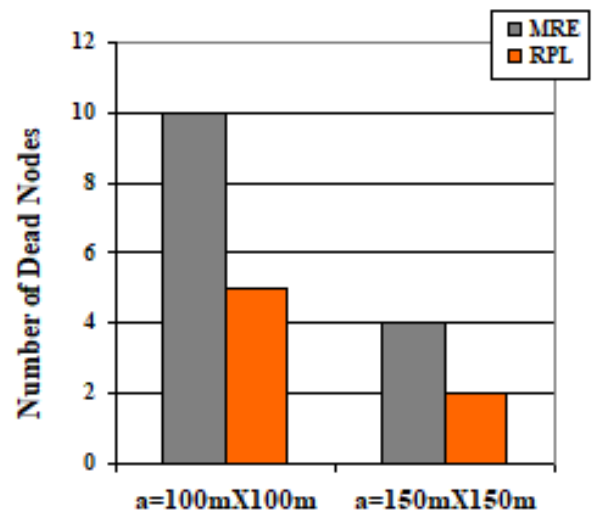

Figure 10: Number of dead nodes for 20 nodes

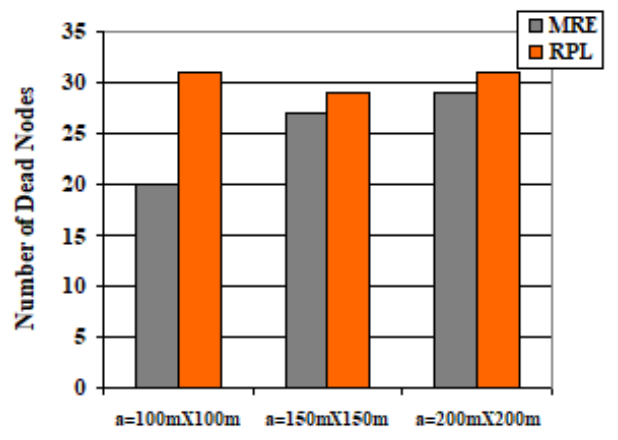

Figure 11: Number of dead nodes for 40 nodes 


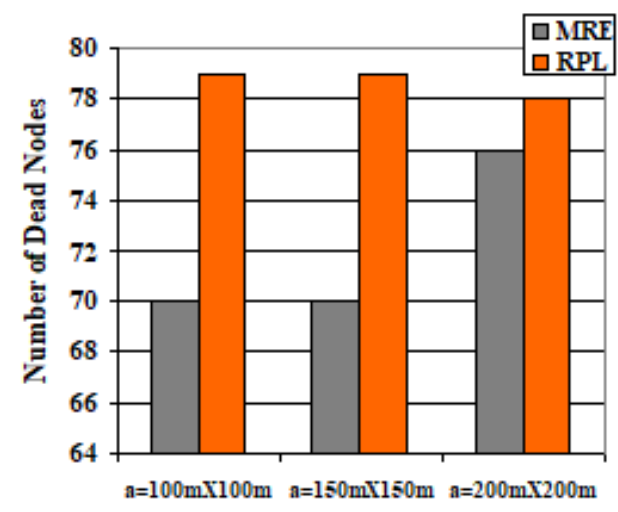

Figure 12: Number of dead nodes for 80 nodes

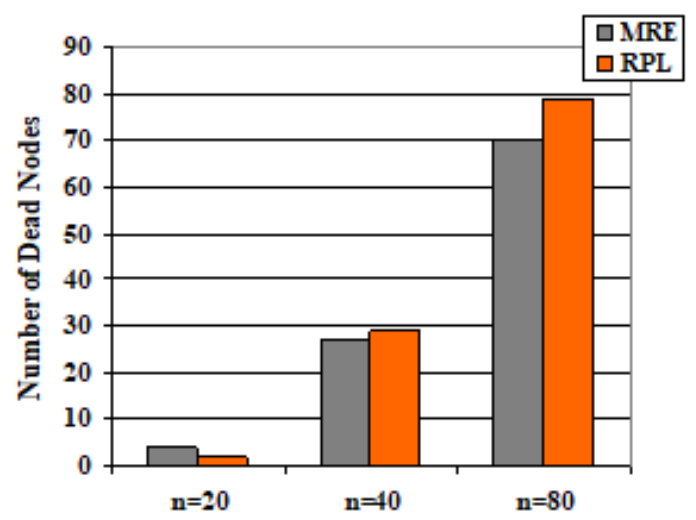

Figure 13: Number of dead nodes for $150 m \times 150 m$ area

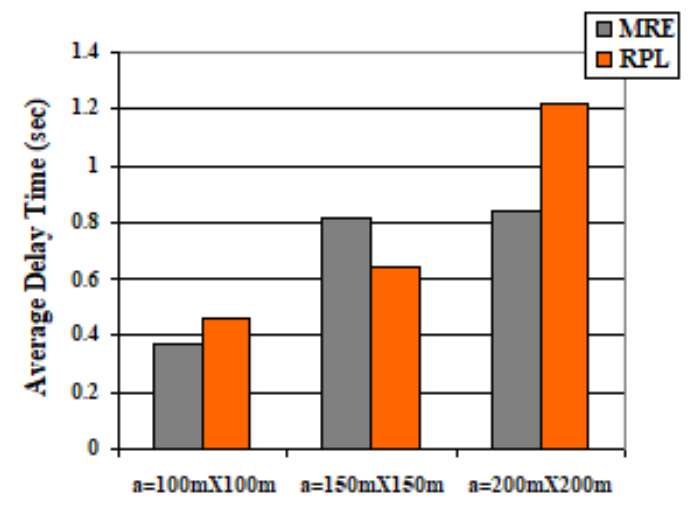

Figure 14: Average delay time for 40 nodes 


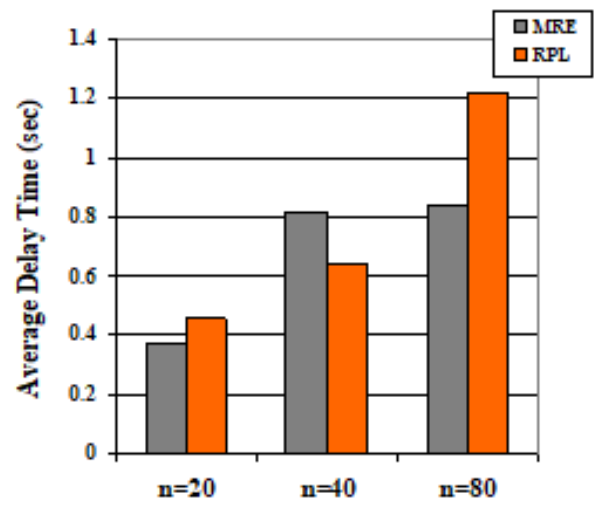

Figure 15: Average time delay for $150 \mathrm{~m} \times 150 \mathrm{~m}$ area

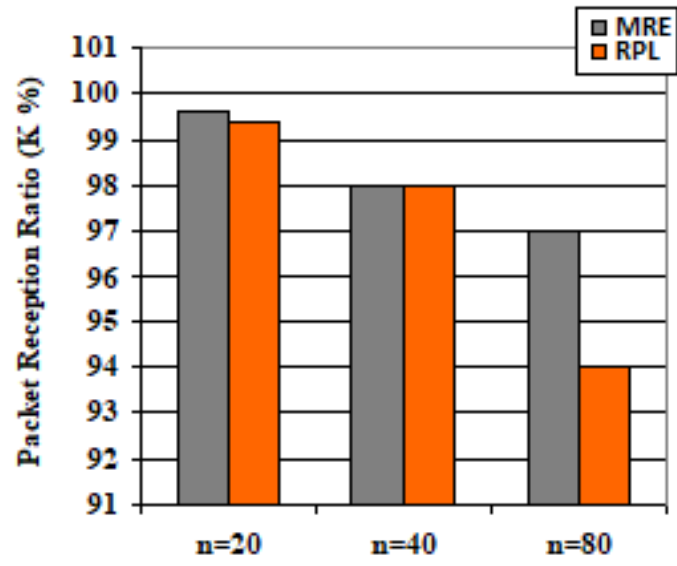

Figure 16: Packet reception ratio for $150 m \times 150 m$ area

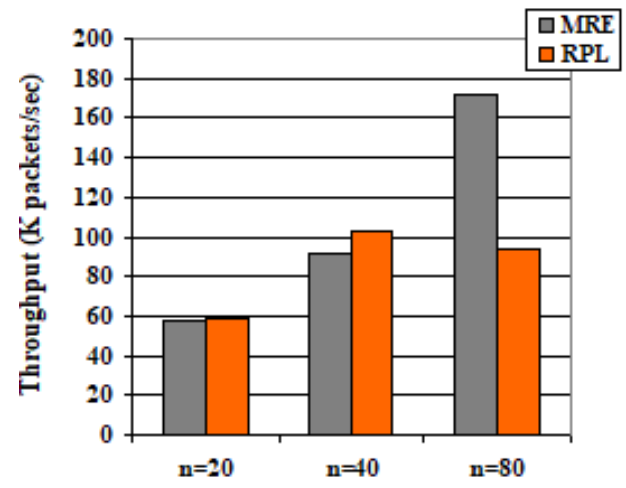

Figure 17: Throughput for $150 m \times 150 m$ area 


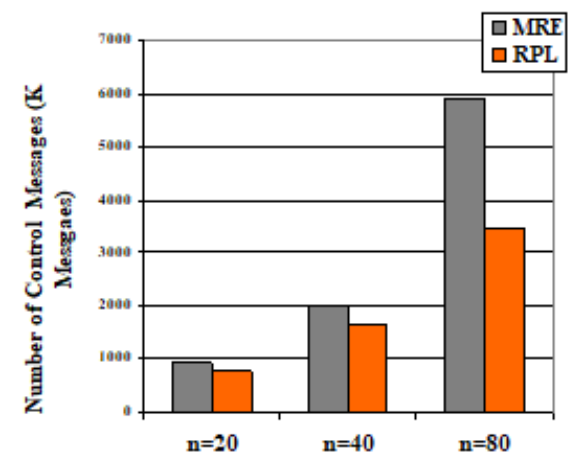

Figure 18: Number of control messages for $150 m \times 150 m$ area

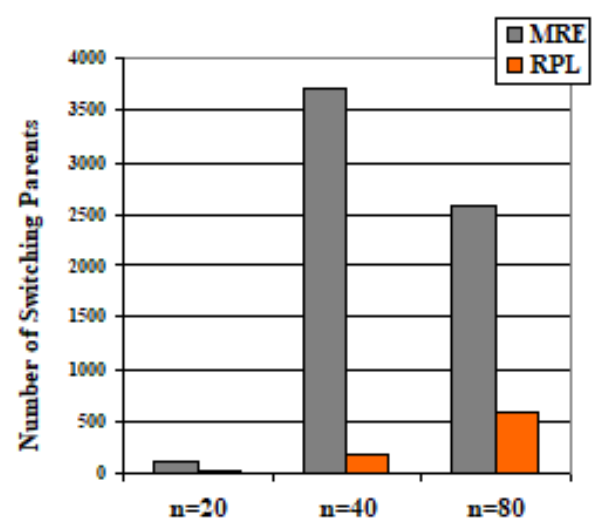

Figure 19: Number of switching parent for $150 m \times 150 m$ area

\section{Conclusions}

The main intension of this paper is to investigate the effect of the neighboring nodes metric combined with the remaining energy of nodes on RPL performance. A Maximum Number of Parent ( MNP) objective function is proposed based on Neighboring metric (N- metric). The proposed MNP based Remaining Energy ( MRE) protocol is tested and compared to the standard RPL. The implementation of both MRE and RPL performed using Cooja simulator with different test conditions. The results showed that MRE achieved more average remaining energy by about $94 \%$ compared to RPL in large network. On the other hand, there is about 33\% loss in average remaining energy in small network. In terms of dead nodes, average delay, and PRR, MRE performed better than RPL when the number of nodes increased in the network. RPL experienced higher average delay compared to MRE by about $31 \%$ in large area. The proposed MRE protocol produced a higher number of control messages and switching parents compared to RPL in all test conditions. Finally, the proposed MRE protocol is useful in saving more energy in large network on the expense of higher switching nodes which may affect the network stability. 


\section{REFERENCES}

[1] M. Abdulrazzaq and A. Kadhim, " Efficient Routing Techniques for Wireless Sensor Networks", Journal of Applied Sciences, vol. 14, no. $24,2014$.

[2] O. Gnawali, and P. Levis, " The Minimum Rank with Hysteresis Objective Function" , IETF, RFC 6719, September 2012.

[3] P. Thubert, " Objective Function Zero for the Routing Protocol for Low-Power and Lossy Networks ( RPL) ", IETF, RFC 6552, March 2012.

[4] Z. WANG, W. Li, and H. Dong, " Analysis of Energy Consumption and Topology of Routing Protocol for Low- Power and Lossy Networks" , IOP Conference Series: Journal of Physics Conference Series ( J Phys Conf), vol. 1087, no. 5, September 2018.

[5] O. Iova, F. Theoleyre, and T. Noel, " Improving Network Lifetime with RPL Through Energy Efficient Load Balancing", IEEE. 7th IFIP Wireless and Mobile Networking ( WMNC), Villamoura, Portugal, May. 2017.

[6] S. Kharche and S. Pawar, " Stability model for RPL with minimum rank hysterisis objective function in 6LoWPAN" , International Journal of Pure and Applied Mathematics, vol. 118, no. 5, Special Issue, pp. 731- 737, 2018.

[7] Kheaksong, A. Srisomboon, A. Prayote, and W. Lee, " Multicriteria parent selection using cognitive radio for RPL in smart grid network" , Wireless Communications and Mobile Computing Journal, pp. 1- 13 [ online] https://www.hindawi.com/journals/wcmc/2018/9590576/ ( accessed 30 December 2019 ).

[8] S. Rafea, " An Efficient Routing Protocol for Energy Management in WSN- Assited IoT" , M. Sc. Thesis, Department of Networks Enginerring, AlNahrain University, Baghdad, Iraq, 2019.

[9] A. Dhumane, R. Prasad, and J. Prasad, " Routing Issues in Internet of Things: A Survey" , International MultiConference of Engineers and Computer Scientists, Hong Kong, March 2016.

[10] R. Poluru and S. Naseera, " A literature review on routing strategy in the internet of things", J. Eng. Sci. Technol. Rev. , vol. 10, no. 5, pp. 5060, 2017.

[11] T. Winter and et al, " RPL: IPv6 Routing Protocol for Low- Power and Lossy Networks" , IETF, RFC6550, pp. 1- 157, March 2012.

[12] J. Vasseur, M. Kim, K. Pister, N. Dejean and D. Barthel, " Routing Metrics Used for Path Calculation in Low Power and Lossy Networks" , IETF, RFC 6551, March 2012.

[13] H. Araujo, R. Filho, J. Rodrigues, R. Rabelo, N. Sousa, J. Filho, and J. Sobral, " A Proposal for IoT Dynamic Routes Selection Based on Contextual Information", Sensors Journal, vol. 18, no. 2. pp. 1- 16, 2018.

[14] A. Velinov and A. Mileva, " Running and Testing Applications for Contiki OS Using Cooja Simulator" , International Conference on Information Technology and Development of Education- ITRO, Zrenjanin, Serbia, 2016.

[15] Z. Wang, W. Li, and H. Dong, " Review on Open Source Operating Systems for Internet of Things" , IOP Conference Series: Journal of Physics Conference Series ( J Phys Conf), vol. 887, no. 1, August 2017.

[16] A. Hicham, A. Sabri, A. Jeghal, and H. Tairi, " A Comparative Study between Operating Systems for the Internet of Things ( IoT) ", Transactions on Machine Learning and Artificial Intelligence/ Special Issue : 1st International Conference on Affective computing, Machine Learning and Intelligent Systems, vol. 5, no. 4, 2017.

[17] M. Yousaf, " Analysis of Simulator Feasibility in Development of Wireless Sensor Network Applications" , M. Sc. Thesis, Department of Communication Systems and Networks, Tampere University of Technology, Hervanta, Finland, 2017.

[18] Moteiv Corporation, " Tmote Sky: Low Power Wireless Sensor Module [ Datasheet] " , pages 28, Nov. 2006, Available: https://insense.cs.st-andrews.ac.uk/files/2013/04/tmote-sky-datasheet.pdf. 